\title{
Prevalence and Factors Associated with Reproductive Tract Infections in Gongolia Village, Rupandehi District, Nepal
}

\author{
Pravina Kafle $^{1}$ and Shiva Shankar Bhattarai ${ }^{2}$ \\ ${ }^{1}$ Department of Public Health, Pokhara University, Lekhnath, Pokhara, Nepal \\ ${ }^{2}$ Department of Horticulture and Plant Protection, Institute of Agriculture and Animal Science, Tribhuvan University, \\ Paklihawa Campus, Rupandehi District, Nepal \\ Correspondence should be addressed to Pravina Kafle; kaphlepraveena20@gmail.com
}

Received 20 June 2016; Revised 1 October 2016; Accepted 3 October 2016

Academic Editor: Ronald J. Prineas

Copyright (c) 2016 P. Kafle and S. S. Bhattarai. This is an open access article distributed under the Creative Commons Attribution License, which permits unrestricted use, distribution, and reproduction in any medium, provided the original work is properly cited.

\begin{abstract}
Objective. To determine the prevalence of reproductive tract infection (RTI) and its associated factors among married women of reproductive age group (15-49 yrs). Method. A cross sectional approach was employed. The study collected data of symptoms suggestive of RTI experienced in the previous six months according to World Health Organization (WHO) syndromic approach guideline. A structured interviewer administered questionnaire was used to collect data and data were entered into EPI data and transferred into SPSS (16 version) for further analysis. Chi square was used for statistical analysis. Result. Among 258 married women the prevalence of symptoms suggestive of RTI was found to be $39.9 \%$. Low back pain, vaginal discharge, and low abdominal pains were common symptoms reported in study. Irregular menstrual cycle, sexual contact during menstruation, marriage below $19 \mathrm{yrs}$, pregnancy below $20 \mathrm{yrs}$, high parity, delivery at home, and use of temporary family planning device were significant risk factors for prevalence of RTI symptoms. Conclusion. RTI still remains neglected by married women. Training and education session needs to be carried out to identify the early symptoms of RTI. Awareness about marriage and pregnancy after $19 \mathrm{yrs}$ of age, sex education, delivery at health institution by health personnel, menstrual and personnel hygiene, and use of the condom are needed for prevention of RTI.
\end{abstract}

\section{Introduction}

Reproductive tract infection (RTI) is defined as the infection of the reproductive or genital tract which causes healthy life loss among sexually active women of reproductive age in developing countries [1]. Women in the reproductive age group are at risk of RTI during natural events in their life such as menstruation, pregnancy, and childbirth [2]. A woman with RTI can represent various symptoms like backache to lower abdominal pain, genital ulcers, vulval itching, inguinal swelling, and abnormal vaginal discharge. RTIs if left untreated can lead to complications like pelvic inflammatory disease (PID), infertility, cervical cancer, chronic pelvic pain, ectopic pregnancy, and pregnancy wastage $[1,3]$.

World Health Organization estimates that over 200 million reproductive tract infections occur due to sexually transmitted pathogens each year among women in developing countries [4]. Despite this significant figure Global Burden of Disease Study estimates that $27.4 \%$ of Disability-Adjusted Life Year lost in Indian women aged 15-44 years was attributed to reproductive ill-health [5]. In Bangladesh study showed that $72.6 \%$ reported one or more symptoms of RTI [6].

In 1996 the Ministry of Health developed reproductive strategies with emphasis on reproductive tract infection and also delivered health services at grass root level. Nepal is signatory to International Conference on Population and Development which aims to achieve universal access to reproductive health by 2015 . In Nepal services related to prevention and management of reproductive tract infections are provided [7].

In Nepal burden of the RTI among women is unexpectedly high. The prevalence of RTI symptom is $78.9 \%$ [8]. The fact is that in Nepal still the RTI burden is presumed to be high. However, data on RTI prevalence are rarely available in 
Nepal [9]. Research and interventions on RTI among women of reproductive age group had been given a high priority in Nepal but data on RTI was still minimally available in Rupandehi District. Therefore, the main aim of this study was to assess prevalence of RTI and its associated factors among married women of reproductive age group (15-49 yrs) in Gongolia village, Nepal.

\section{Materials and Methods}

2.1. Study Area and Period. The study was conducted in Gongolia village of Rupandehi District. The data were collected from November to December 2014.

2.2. Design and Study Population. A community based cross sectional study was conducted. Married women of reproductive age group (15-49 yrs) residing in selected ward of Gongolia village were included in the study.

2.3. Sample Size Determination. The sample size was determined by using single population proportion formula considering prevalence of reproductive tract infection as $27 \%$ from previous study in Kathmandu [3], margin of error as $0.7 \%$, and $95 \%$ as confidence interval and 1.55 design effects. After including 10\% nonresponse rates, 258 married women of reproductive age group were included in the study.

2.4. Sampling Method. Cluster sampling technique was used in this study. Interviewed married women were selected from four wards which were selected by using random method from all wards. Then the numbers of study participants were determined proportionately from selected ward by using community based demographic registration prepared by Village Development Committee as the sampling frame. After that respondents to be interviewed were selected randomly.

2.5. Data Collection Tool. A structured interviewer administered questionnaire was used to collect data. The questionnaire was prepared in English language, and then it was translated into a local language.

2.6. Data Processing and Analysis. Data were entered into EPI data and transferred into Statistical Package for Social Science (SPSS-16 version) for editing and further analysis. Based on objectives the study data were analyzed. The data was analyzed by using descriptive and inferential statistics. Descriptive statistics (frequency, percentage, mean, median, and standard deviation) and inferential statistics (chi square) were applied to perform required statistical output.

2.7. Ethical Consideration. The study obtained ethical approval from public health program of School of Health and Allied Sciences. Informed consent from each study participant was obtained after clear explanation about the purpose of the study. Confidentiality and privacy of data were maintained.

2.8. Inclusion and Exclusion Criteria. Married women who are available at the time of data collection were included and
TABLE 1: Prevalence of symptoms suggestive of reproductive tract infections.

\begin{tabular}{lcc}
\hline Prevalence of RTI & Frequency & Percentage \\
\hline Yes & 103 & 39.9 \\
No & 155 & 60.1 \\
Total & 258 & 100 \\
\hline
\end{tabular}

TABLE 2: Distribution of symptoms suggestive of RTI among married women experienced in last six months $(n=258)$.

\begin{tabular}{lcc}
\hline Symptoms & Frequency & Percentage \\
\hline Backache & 84 & 32.6 \\
Vaginal discharge & 69 & 26.7 \\
Low abdominal pain & 50 & 19.4 \\
Itching around vaginal area & 41 & 15.9 \\
Painful or burning urination & 27 & 10.5 \\
Painful intercourse & 16 & 6.2 \\
Genital ulceration & 6 & 2.3 \\
Inguinal swelling & 0 & 0 \\
\hline
\end{tabular}

only menstruation related questions were not asked to those women who are pregnant and menopausal during data collection time.

\section{Result}

The prevalence of symptoms of RTI among married women of reproductive age in the study population was found to be $39.9 \%$. The research found that mean age of respondent was $30.95 \pm 7.579$. World Health Organization (WHO) syndromic approach guideline was considered for recognizing the symptoms suggestive of RTI. During study period eligible women were asked about whether they had experienced any suggestive symptoms of RTI in the last six months. If participant had experienced at least two symptoms then they are considered to have RTI. Among 258 eligible married women $39.9 \%$ had experienced at least two or more than two symptoms of RTI in the past six months which was shown on Table 1. Most common experienced symptoms suggestive of RTI were low back pain, 32.6\%, followed by vaginal discharge, $26.7 \%$, low abdominal pain, $19.4 \%$, itching around vagina, $15.9 \%$, painful or burning urination, $10.5 \%$, painful intercourse, $6.2 \%$, and genital ulceration, $2.3 \%$, while no one had symptom of inguinal swelling which had been shown on Table 2.

3.1. Factors Associated with Symptoms Suggestive of RTI. In this present study sociodemographic variables were not associated factors for RTI shown on Table 3. Similarly irregular menstrual cycle and sexual contact during menstruation were risk factors shown on Table 4. Moreover marriage below 19 years of age, pregnancy below 20 years of age, delivery at home, and delivery conducted by non-health personnel were found to be significantly associated with RTI which was shown on Table 5. 
TABLE 3: Sociodemographic related factors $(n=258)$.

\begin{tabular}{lcccc}
\hline Variables & Had RTI & No RTI & $\chi^{2}$ & $p$ value \\
\hline $\begin{array}{l}\text { Age of respondent } \\
<30\end{array}$ & $54(38.6)$ & $86(61.4)$ & 0.233 & 0.629 \\
$\geq 30$ & $49(41.5)$ & $69(58.5)$ & & \\
Educational status & & & & \\
Illiterate & $27(38.0)$ & $44(62.0)$ & 2.999 & 0.22 \\
Informal education & $41(47.1)$ & $46(52.9)$ & & \\
Formal education & $35(35.0)$ & $65(65.0)$ & & \\
$\begin{array}{l}\text { Occupation } \\
\text { Business/external job }\end{array}$ & $9(40.9)$ & $13(59.1)$ & 0.10 & 0.92 \\
Housework & $94(39.8)$ & $142(60.2)$ & & \\
Monthly income & & & & \\
$<10000$ & $78(41.9)$ & $108(58.1)$ & 7.969 & 0.289 \\
$\geq 10000$ & $25(34.7)$ & $47(65.3)$ & & \\
Type of family & & & & \\
Nuclear & $28(41.8)$ & $39(58.2)$ & 0.132 & 0.717 \\
Joint & $75(39.3)$ & $116(60.7)$ & & \\
\hline
\end{tabular}

Figure in parenthesis indicates percentage, formal education: primary level to bachelor level, housework: agriculture \& housewife, informal education (literate).

In this present study among 258 married women 17 were are not applicable for asking menstrual related questions because 14 were currently pregnant and 3 women's menstruation was stopped. Having irregular menstruation cycle, sexual intercourse during menstruation, and bathing once a day during period were risk factors associated with RTI.

\section{Discussion}

4.1. Symptomatic Prevalence of Reproductive Tract Infection. The aim of the study was to find out the prevalence and factors associated with RTI among married women of reproductive age group (15-49 yrs). From the study the prevalence of symptoms suggestive of RTI was found to be $39.9 \%$. Prevalence was reported to be 15 percent to 80 percent in similar community based studies [1,3,8,10-13]. Study finding somehow agrees with Mani et al. $[1,10,13]$. Similarly Dameru et al. observed higher prevalence than present study; it might be due to difference in study area and population $[3,8]$. On the other hand, Philip et al. marked prevalence of RTI quite lower than the present study $[11,12]$.

This study revealed that distribution of symptoms suggestive of RTI reported by married women was low back pain, $32.6 \%$, followed by vaginal discharge, $26.7 \%$, low abdominal pain, $19.4 \%$, itching around vaginal area, $15.9 \%$, painful or burning urination, $10.5 \%$, painful intercourse, $6.2 \%$, genital ulceration, $1.9 \%$, and warts around vaginal and anus area, $0.4 \%$. Similarly Dameru et al. have reported identical symptoms, that is, vaginal discharge, lower abdominal pain, low backache, vaginal itching and pain during urination, and painful intercourse, which are consistent with the current finding $[8,12,14]$.
4.2. Risk Factors of RTI among Married Women of Reproductive Age Group. A report of this study revealed that prevalence of symptoms suggestive of RTI marked higher in women who are aged 30 yrs or above. However, a study reported by Dameru et al. showed that age of 30 yrs and above had significant association with outcome variables $[8,11]$. In this observation women having informal education had more RTI cases. This finding is in line with a study done by Dameru [8].

This study indicated that numerically more numbers (41.9\%) of participants with RTI had monthly income of NRs $<10000$ where income was not a significant factor of RTI. A study reported by Dameru et al. showed that living in low and middle income socioeconomic status was risk factor for RTI symptoms $[8,15]$.

On this finding about $41.8 \%$ of symptoms suggestive of RTI were found in nuclear family which was not a significant factor of RTI symptom. A study done in Ludhiana [11] showed lower prevalence of RTI in nuclear family (15.4\%).

The finding of study showed that more than half of married women who had symptoms suggestive of RTI had irregular menstrual cycle. On statistical analysis it was found that having irregular menstrual cycle is significantly associated with outcome variables. This finding was supported by the study conducted in Kaski District by Dameru [8].

This study showed that women using cloth during menstruation have high prevalence of RTI. This finding was also supported by Thekdi et al. who reported that using cloth during period was a significant factor for RTI [3]. Likewise, the present study showed that there was lower prevalence of RTI symptoms among women who take bath once a day during menstruation that is good hygiene practice. Prevalence of RTI symptoms was observed to be five and more times more among those who had sexual intercourse during menstruation as compared to those who did not. This finding was similar to Dameru who reported that sexual intercourse during menstruation was highly significantly associated with outcome variables [8].

On statistical analysis it was found that those women married below 19 years of age are likely to have $43.3 \%$ RTI symptoms as compared to those married at or above 19 years of age. These findings are in line with another study by Parashar et al. [13] conducted in Shimla city. Early age at marriage means early sexual activities, which may cause trauma, hence offering a platform for future infections.

There was strong statistical association between RTI symptoms and age of pregnancy below $20 \mathrm{yrs}$. Prevalence of RTI symptoms was $46.4 \%$ among women who had pregnancy at below 20 years as compared to above and at 20 years. A higher prevalence of RTI symptoms was found, about $42.7 \%$, among women who had three or more children and it increased with increase in parity. This finding was consistent with Parashar et al. who reported that having three children or more showed higher prevalence of RTI [13].

The present study showed that women who gave birth at their own home and conducted delivery by non-health personnel are at significant risk of RTI symptoms. There was also strong association between RTI symptoms and using of contraception. The study conducted by Parashar et al. in Simla city [13] is in line with this present study. 
TABLE 4: Menstrual hygiene related factors $(n=241)$.

\begin{tabular}{|c|c|c|c|c|}
\hline Variables & Had RTI & No RTI & $\chi^{2}$ & $p$ value \\
\hline \multicolumn{5}{|l|}{ Menstruation cycle } \\
\hline Irregular & $30(57.7)$ & $22(42.3)$ & 7.969 & $0.005^{* *}$ \\
\hline Regular & $68(36.0)$ & $121(64.0)$ & & \\
\hline \multicolumn{5}{|c|}{ Material used during menstruation } \\
\hline Sanitary pad & $8(47.1)$ & $9(52.59)$ & 0.310 & 0.578 \\
\hline Cloth or both & $90(40.1)$ & $134(59.8)$ & & \\
\hline \multicolumn{5}{|c|}{ Frequency of changing pad/cloth } \\
\hline Once a day & $28(40.0)$ & $42(60.0)$ & 0.18 & 0.893 \\
\hline Twice a day or more & $70(40.9)$ & $101(59.1)$ & & \\
\hline \multicolumn{5}{|c|}{ Frequency of bath during menstruation } \\
\hline Once a day & $48(38.1)$ & $78(61.9)$ & 2.123 & 0.346 \\
\hline Twice a day & $19(51.4)$ & $18(48.6)$ & & \\
\hline Thrice a day & $31(39.7)$ & $47(60.3)$ & & \\
\hline \multicolumn{5}{|c|}{ Sexual contact during menstruation } \\
\hline Yes & $22(75.9)$ & $7(24.1)$ & 16.92 & $0.001^{* *}$ \\
\hline No & $76(35.8)$ & $136(64.2)$ & & \\
\hline
\end{tabular}

Figure in parenthesis indicates percentage, ${ }^{* *}$ statistically highly significant at $p<0.01$.

TABLE 5: Marital and reproductive status as risk factors for RTI $(n=258)$.

\begin{tabular}{|c|c|c|c|c|}
\hline & RTI (yes) & RTI (no) & $\chi^{2}$ & $p$ value \\
\hline \multicolumn{5}{|l|}{ Age at marriage } \\
\hline$<19$ & $98(43.3)$ & $130(57.0)$ & 7.655 & $0.006^{* *}$ \\
\hline$\geq 19$ & $5(16.7)$ & $25(83.3)$ & & \\
\hline \multicolumn{5}{|l|}{ Age at first pregnancy } \\
\hline$<20$ & $89(46.4)$ & $103(53.6)$ & 8.142 & $0.004^{* *}$ \\
\hline$\geq 20$ & $13(24.5)$ & $40(75.5)$ & & \\
\hline \multicolumn{5}{|l|}{ Parity } \\
\hline None & $1(6.7)$ & $14(93.3)$ & 7.398 & $0.025^{*}$ \\
\hline $1-2$ & $52(41.3)$ & $74(58.7)$ & & \\
\hline$\geq 3$ & $50(42.7)$ & $67(57.3)$ & & \\
\hline \multicolumn{5}{|l|}{ Place of delivery } \\
\hline Home & $67(48.2)$ & $72(51.8)$ & 4.527 & $0.023^{*}$ \\
\hline Health institution & $35(33.7)$ & $69(66.3)$ & & \\
\hline \multicolumn{5}{|l|}{ Delivery assistant } \\
\hline Non-health personnel & $65(48.1)$ & $70(51.9)$ & 4.814 & $0.029^{*}$ \\
\hline Health personnel & $37(34.3)$ & $71(65.7)$ & & \\
\hline \multicolumn{5}{|l|}{ Conduct abortion } \\
\hline Yes & $2(66.7)$ & $1(33.3)$ & 0.851 & $0.565^{+}$ \\
\hline No & $101(39.6)$ & $154(60.4)$ & & \\
\hline \multicolumn{5}{|l|}{ Use of family planning device } \\
\hline Yes & $76(48.1)$ & $82(51.9)$ & 11.195 & $0.001^{* *}$ \\
\hline No & $27(27.6)$ & $73(73.0)$ & & \\
\hline \multicolumn{5}{|l|}{ Type of family planning device } \\
\hline Temporary family planning & $51(50.3)$ & $50(49.5)$ & 0.643 & 0.42 \\
\hline Permanent family planning & $25(43.9)$ & $32(56.1)$ & & \\
\hline
\end{tabular}

Figure in parenthesis indicates percentage, ${ }^{*}$ statistically significant at $p<0.05,{ }^{* *}$ statistically highly significant at $p<0.01,{ }^{+}$fisher exact test, temporary (condoms, depo, IUD, pills). 


\section{Conclusion}

A total of 39.9\% married women had prevalence of symptoms suggestive of RTI experienced in last six months. The most common symptoms reported by the women sufferers were low back pain followed by vaginal discharge, low abdominal pain, itching around vaginal area, painful or burning urination, painful intercourse, and genital ulceration. Irregular menstrual cycle, sexual contact during menstruation, marriage at below 19 years, pregnancy below 20 years, delivery at home, delivery and conducted by non-health personnel were significant risk factors for prevalence of RTI. Training and education session needs to be carried out to identify the early symptoms of RTI. Awareness about marriage and pregnancy after 19 yrs of age, sex education, delivery at health institution by health personnel, menstrual and personnel hygiene, and use of the condom are needed for prevention of RTI.

\section{Competing Interests}

The authors declare that there is no conflict of interests regarding the publication of this paper.

\section{Acknowledgments}

Authors are grateful to the Department of Public Health, School of Health and Allied Sciences, Pokhara University, Lekhnath, Kaski, for their permission to conduct research and support. The authors salient gratitude goes to the District Public Health Office, Rupandehi and Gongolia Village Development Committee, for granting approval to conduct research. Special thanks go to Shukriti Bhandari, Sadiksha Regmi, and whole respondents who cooperated, were supportive, and gave their personal information openly.

\section{References}

[1] R. P. Ravi and R. A. Kulasekaran, "Care seeking behaviour and barriers to accessing services for sexual health problems among women in rural areas of tamilnadu state in India," Journal of Sexually Transmitted Diseases, vol. 2014, Article ID 292157, 8 pages, 2014.

[2] M. Geetha, "Prevalence of reproductive tract infections among rural married women in Tamil Nadu, India: a community based study," Journal of Pioneering Medical Sciences, vol. 4, no. 1, pp. 18-24, 2014.

[3] K. P. Thekdi, N. K. Patel, K. G. Patel, and P. I. Thekdi, "Health seeking attitude of women regarding reproductive tract infections in a rural area of Surendranagar district," International Journal of Research in Medical Sciences, vol. 1, no. 4, pp. 552-556, 2013.

[4] World Health Organization, Global Prevalence and Incidence of Selected Transmitted Infection, Overview and Estimate, WHO, Geneva, Switzerland, 2001.

[5] J. Bhatia and J. Cleland, "The contribution of reproductive illhealth to the overall burden of perceived illness among women in southern India," Bulletin of the World Health Organization, vol. 79, no. 11, pp. 1065-1069, 2001.

[6] S. M. E. Ashikur, M. Saleh, T. Sezan, and Z. Raham, "Breaking the silence: reproductive tract infections (RTIs) among women in slums of Khulna city, Bangladesh," Bangladesh E-Journal of Sociology, vol. 10, no. 2, pp. 119-134, 2013.

[7] National Reproductive Health Commodity Security (2007-2011), Government of Nepal, Department of Health Services, Ministry of Health and Population, 2006.

[8] P. P. Dameru, "Prevalance and factor associated with reproductive tract infection among married women of reproductive age in Kaski district, Nepal," Asian Journal of Medical Science, vol. 2, no. 1, pp. 46-49, 2012.

[9] Institute of Medicine/United National Children Fund, Status of Reproductive Morbidity in Nepal (A Research Report Submitted to UNEFA), Institute of Medicine, Kathmandu, Nepal, 2006.

[10] G. Mani, K. Annadurai, and R. Danasekaran, "Healthcare seeking behaviour for symptoms of reproductive tract infections among rural married women in Tamil Nadu-a community based study," Online Journal of Health and Allied Sciences, vol. 12 , no. 3, article 3, 2013

[11] P. S. Philip, A. I. Benjamin, and P. Sengupta, "Prevalence of symptoms suggestive of reproductive tract infections/sexually transmitted infections in women in an urban area of Ludhiana," Indian Journal of Sexually Transmitted Diseases, vol. 34, no. 2, pp. 83-88, 2013.

[12] S. Yasmin and A. Mukherjee, "A cyto-epidemiological study on married women in reproductive age group (15-49 years) regarding reproductive tract infection in a rural community of West Bengal," Indian journal of public health, vol. 56, no. 3, pp. 204-209, 2012.

[13] A. Parashar, B. Gupta, A. Bhardwaj, and R. Sarin, "Prevalence of RTIs among women of reproductive age group in Shimla city," Indian Journal of Community Medicine, vol. 31, no. 1, pp. 15-17, 2006.

[14] K. N. Mamta, "Reproductive tract infection: prevalence and health seeking behavior among women of reproductive age group," International Journal of Science \& Research, vol. 3, no. 4, pp. 138-142, 2014.

[15] Y. K. Kazi, A. G. Shenoy, G. Velhal, and S. R. Suryawanshi, "Reproductive and sexual tract infections among married female youth in an urban slum of Mumbai," National Journal of Community Medicine, vol. 4, no. 1, pp. 10-14, 2013. 


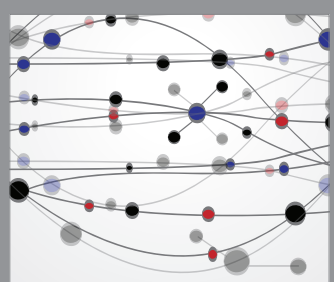

The Scientific World Journal
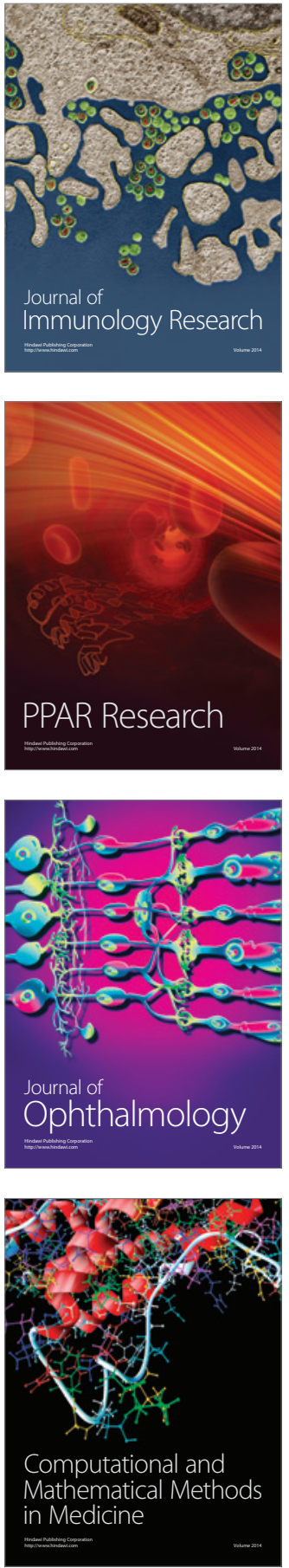

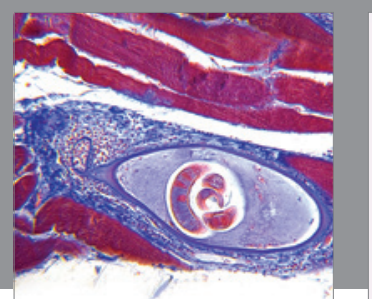

Gastroenterology Research and Practice

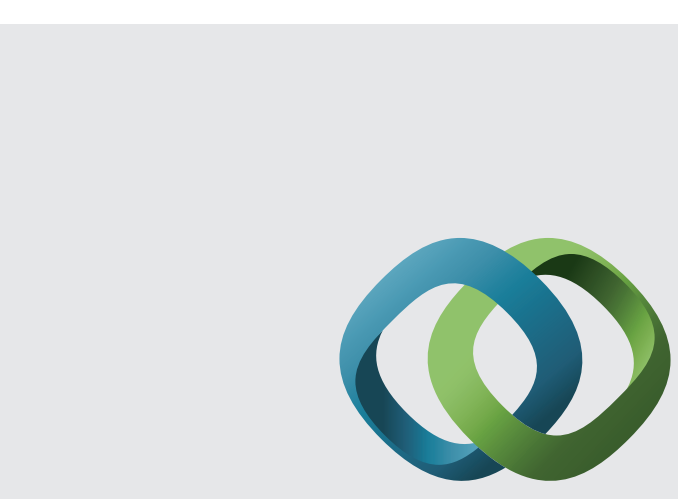

\section{Hindawi}

Submit your manuscripts at

http://www.hindawi.com
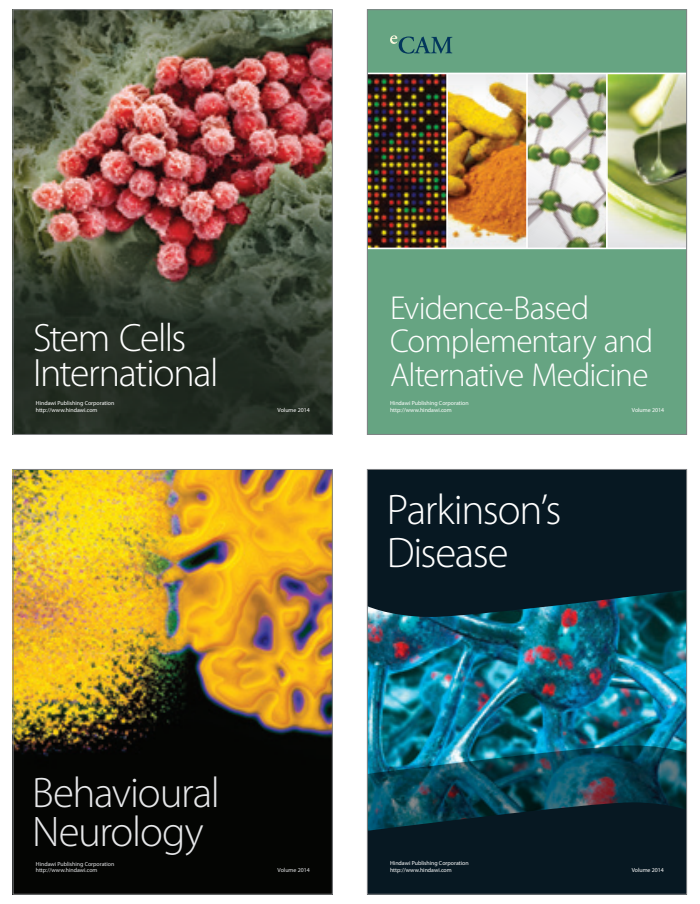
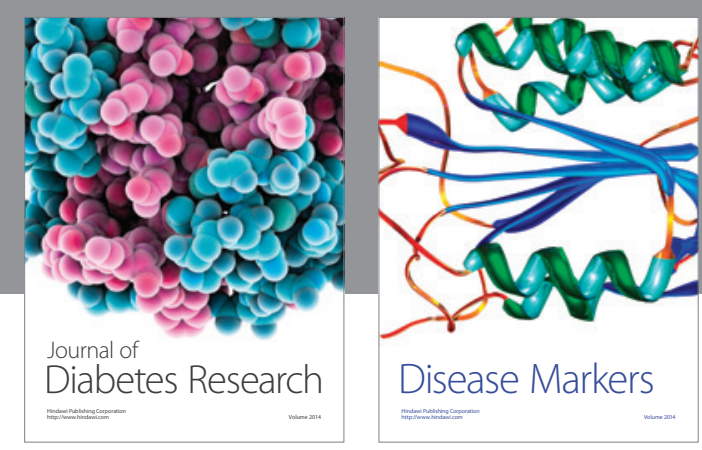

Disease Markers
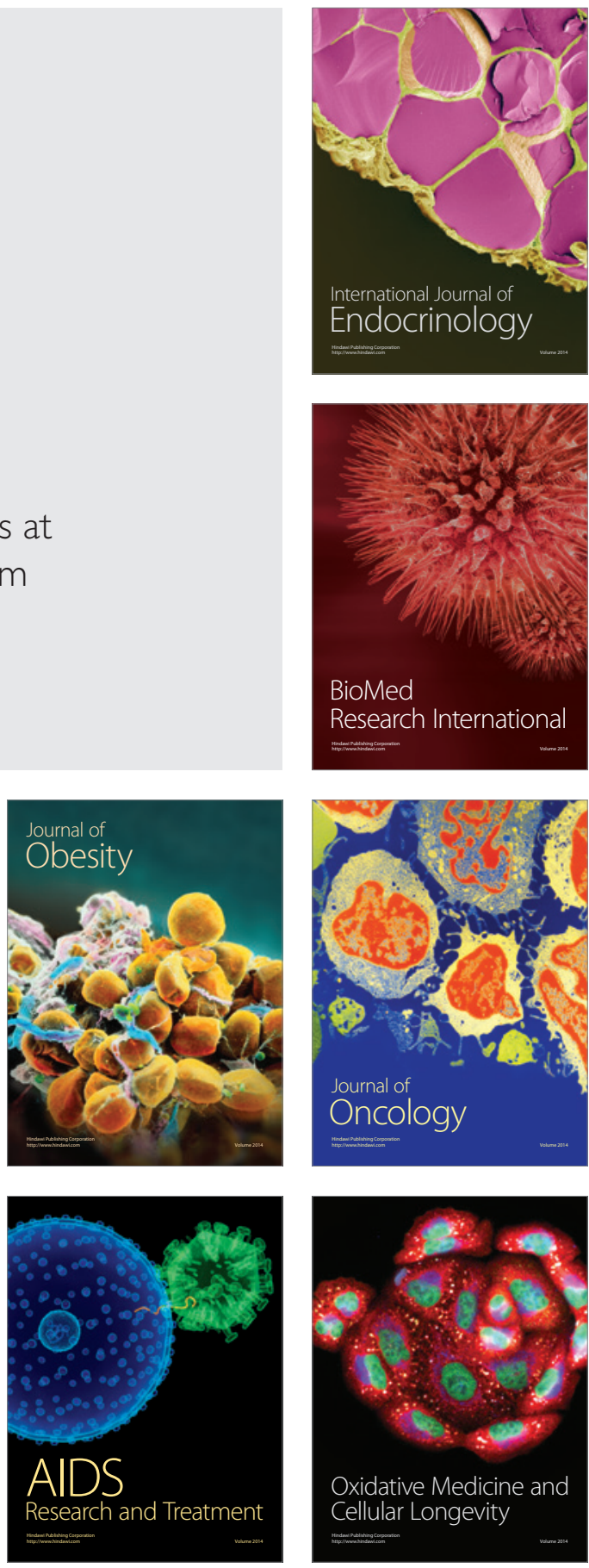